P Gli articoli di questa sezione sono sottoposti a referaggio doppiamente cieco (double blind peer review process) e seguono gli standard in uso per le pubblicazioni scientifiche a livello internazionale ed accettati dalle principali banche dati citazionali

\title{
Carcere e clinica delle dipendenze. Quali le sfide da affrontare per mantenere terapeutico un sistema di cura?
}

\author{
Emanuele Bignamini*, Sara Zazza ${ }^{\circ}$ Loredana Ierardi $\$$, Enrico Teta**
}

\begin{abstract}
SUMMARY
- The "Arcobaleno" facility for mitigated prison custody "Lorusso e Cutugno" Penitentiary in Turin accommodates about 80 patients affected by substance use disorders, capable of joining the provided Community therapeutic program. If, for the penitentiary administration, a prisoner is a detainee, i.e. a person guilty of offences to expiate and who must be supervised and rehabilitated, he/she is a person who needs an articulated medical and psychosocialeducational treatment, according to the Health Service. This different point of view leads to carry out distinct styles of treatment and therapy. It is influenced by substantial and uncontrollable risks and intertwines positive aspects with different problems that have always been present in all approaches. The severe, and sometimes almost untreatable, patients, such as these detainees, cause a strong feeling of loss, helplessness and envy to all of those people who take care of them (health personnel and prison warders), who are forced to constantly process these emotions. How can clinic treatment deal with penitentiary system conditions? Is it possible? After due analysis of relational and context dynamics established within a detention facility, the Authors try to give a first answer to the question concerned.
\end{abstract}

Keywords: Attachment, Aggressiveness, Time, Pedagogy of responsibility, Cost-effectiveness.

Parole chiave: Attaccamento, Aggressività, Tempo, Pedagogia della responsabilità, Costi e benefici.

\section{Il carcere e il mondo esterno}

Il carcere è concepito come luogo chiuso, finalizzato a sottrarre alla vista (senso che mette in contatto con l'ambiente e che ha avuto il sopravvento sugli altri sensi dopo I'acquisizione della stazione eretta) persone che, più di altre, suscitano paura e insicurezza nell'immaginario collettivo, rappresentando l'incarnazione del "male" (Foucault, 1976).

Acquista, dunque, la funzione di separatore: non è un caso, infatti, che venga costruito lontano dal cuore della vita sociale, decentrato da tutto.

La conseguenza di un luogo così concepito, dove diventa facile per chi ci vive estraniarsi dal resto del mondo, è l'impenetrabilità e l'impossibilità di comunicazione con l'esterno, perseguita come un presupposto essenziale. La porta che chiude le sue spesse mura non è una porta girevole, in grado di dialogare e creare ponti con la società, bensì un ingresso che si serra, spa-

* Direttore Struttura Complessa Dipendenze 1, ASL ex TO2, Torino.

- Psicoterapeuta, Servizio Dipendenze, ASL ex TO2, Torino.

$\S$ Educatrice Professionale, Servizio Dipendenze, ASL ex TO2, Torino.

** Referente Servizio Dipendenze - Area Penale, ASL "Città di Torino". lancandosi a un mondo popolato da regole e da logiche certamente diverse e a volte opposte e contrarie a quanto si trova fuori le mura. Di per sé, un luogo chiuso che non si relaziona con l'esterno si perpetua senza mai cambiare, diventando sempre più autoreferenziale e in grado di fagocitare tutto ciò con cui entra in contatto. In un tale ambiente, sopravvive chi riesce a mettere in atto meccanismi di assimilazione.

\section{L'ingresso in carcere e le modalità di attacca- mento}

L'ingresso in carcere è un passaggio carico di stress, destinato inevitabilmente a riattivare i meccanismi di difesa, i modelli di attaccamento acquisiti e le conseguenti strategie di ricerca di conforto. La cesura segnata dall'ingresso in carcere comporta, infatti, la separazione reale e simbolica dalle figure di riferimento significative.

Il comportamento di attaccamento ha la duplice funzione di assicurare la vicinanza a una figura di attaccamento e di proteggere il piccolo dal pericolo (Bowlby, 1996). È la caratteristica principale che dovrebbero offrire i genitori, ossia quella di essere pronti e disponibili a rispondere se chiamati in causa, incorag- 
giare e dare assistenza, limitandosi però a intervenire attivamente solo quando è necessario, senza "iperproteggere".

Il contesto detentivo, proprio per il suo esplicito mandato di "custodire chi è colpevole e deve scontare una pena" e le sue rigide strutturazioni e gerarchie, non può essere certo assimilato a una madre-ambiente responsiva (Winnicott, 2005) e disponibile ai bisogni di conforto e angoscia dei detenuti. L'angoscia non è mitigata dalla vicinanza, che può variare dal semplice "essere visti", alla vicinanza fisica senza contatto ma accompagnata da parole di conforto, fino al vero e proprio essere con-tenuti fisicamente. Gli agenti non possono stabilire legami privilegiati con i detenuti, né si possono permettere relazioni di tipo affettivo. Il rapporto numerico detenuti/agenti cristallizza la finalità primaria custodialistica e repressiva del carcere, negando quella "ridondanza" che permetterebbe l'esplorazione di alternative relazionali. I detenuti, quindi, nel contesto carcerario, sperimentano prevalentemente una sensazione di pericolo e non possono sentirsi protetti da un simile contesto.

La popolazione di detenuti di cui ci occupiamo come Dipartimento Dipendenze sono soggetti portatori sia di problemi con la giustizia sia di patologie da dipendenza.

I detenuti-pazienti di questo tipo propongono di solito, in ordine alle esperienze precoci e ai life events (Ferrero, 1995) un tipo di costruzione del legame che può essere prevalentemente riferito a due modelli di attaccamento: evitante ed ansioso-ambivalente (Liotti e Farina, 2011).

Comune alle due modalità è la relazione di attaccamento insicura, che denota che non si è costruita una sintonia sufficientemente buona tra il bambino e la figura di accudimento: quest'ultima viene dal primo percepita come non disponibile, poco responsiva rispetto alle sue richieste e incapace di mettersi in rapporto con i suoi bisogni. I soggetti insicuri hanno modelli operativi interni poco accurati, centrati sull'idea di dover "essere all'altezza di", in un continuo tentativo di adattamento all'agente delle cure materne. Possono adottare due diverse strategie - I'evitamento o l'adesione - dando vita così a un attaccamento evitante oppure a uno ansioso-ambivalente.

Nell'attaccamento evitante il bambino tenta di minimizzare i propri bisogni di attaccamento per prevenire il rifiuto: rimane in contatto con il caregiver, ma cerca e concede una vicinanza emotiva minima.

La strategia ambivalente comporta, invece, I'aggrapparsi al caregiver con una sottomissione spesso eccessiva, o l'adozione di un'inversione di ruolo, per cui è il bambino a curarsi dell'agente delle cure materne e non viceversa, come dovrebbe ovviamente essere.

\section{I percorsi terapeutici-riabilitativi}

Nella prima fase immediatamente successiva all'ingresso in carcere del detenuto-tossicodipendente, anche in ordine a quanto sopra accennato, I'obiettivo del gruppo curante è fornire riferimenti che permettano alla persona di mantenere una connessione di cura fra l'esterno (trattamento già avviato o da avviare al Servizio Dipendenze territoriale) e l'interno (carcere). Gli operatori del Ser.D. Area Penale afferente al Dipartimento Dipendenze avviano la prima interazione e avviano anche, laddove è necessario, il sostegno farmacologico.

Sulla base della valutazione clinica raccolta e della formalizzazione della diagnosi di dipendenza, potranno poi essere proposte al detenuto-paziente la continuazione del percorso terapeutico all'interno di una Struttura a Custodia Attenuata (SCA) deno- minata "Arcobaleno". Questa SCA, rispetto alla detenzione presso i blocchi ordinari, consente al detenuto, previa la sottoscrizione di un contratto terapeutico impegnativo, una maggiore flessibilità sia di orari sia di spostamenti nella sezione al fine di partecipare al progetto terapeutico, che è articolato in due percorsi: uno rivolto a chi deve essere sostenuto alla richiesta di una misura alternativa alla detenzione, I'altro per chi passa tutto il resto della pena all'interno del carcere. Questo tipo di proposta vede impegnati diversi professionisti della ASL (psicologo, educatore, medico, infermiere, assistente sociale) su un arco temporale di otto ore per cinque giorni la settimana. II tempo, lo spazio e le finalità generali, nonché alcune attività specifiche sono condivisi con il personale di Polizia Penitenziaria (Ispettore e Agenti) e con il personale civile dell'Amministrazione Penitenziaria (educatori e conduttori di laboratori).

All'interno della SCA si confrontano, quindi, il sistema penitenziario e quello sanitario, portatori di due culture differenti e l'esito in ordine al benessere e alla qualità emotiva del sistema è, dunque, il risultato del confronto di queste due culture.

Se la persona reclusa è, per l'Amministrazione Penitenziaria, un detenuto, ovvero un soggetto reo di colpe da scontare e che va controllato e rieducato, per il Servizio Sanitario è una persona che richiede un trattamento articolato, sanitario e psicosocioeducativo. Questa differenza di sguardo porta a mettere in atto stili "trattamentali" o "terapeutici" diversi e reciprocamente influenzantesi; ovviamente, si auspica che "I'integrazione" avvenga solo per gli aspetti positivi delle parti diverse, e che il sistema nel suo complesso proceda verso il "bene". Tuttavia esistono rischi consistenti e poco controllabili (certamente non controllabili in tempo reale e senza costi) che invece si integrino le diverse problematiche, sempre presenti in ognuno degli approcci.

Il primo sguardo persegue la riabilitazione attraverso una rieducazione del comportamento deviante: la tossicodipendenza e il reato sono visti come un comportamento errato che verrà abbandonato dopo aver appreso una nuova scala valoriale come guida per la "nuova" vita e nuove "abilità". Le regole, in questa cornice, sono scelte sulla base di un "ideale di vita" prefigurato come "buono e giusto". Non sono, quindi, negoziabili e la persona deve adeguarvisi per dimostrare sia il proprio cambiamento, sia la propria motivazione a rimanere all'interno del percorso rieducativo. Ovviamente, la "verifica" non può che basarsi su elementi osservabili dall'esterno e non ha accesso al "cambiamento interiore", alla metanoia della persona. Il messaggio trasmesso è che bisogna aderire a ciò che il contesto impone, congelando la persona (il paziente, ma anche l'operatore) a rispettare delle regole che alimentano una morale preconvenzionale (Piaget, 1972).

La moralità coincide, cioè, con l'ubbidienza a chi ha autorità. Eventuali eccezioni sono avvertite come pericolose, minacciose per la credibilità e la stabilità del sistema trattamentale, potenzialmente ingiuste in quanto introducono differenze tra i detenuti che contraddicono il dichiarato "siamo tutti uguali". Se contemplate, le eccezioni assumono un carattere di "concessione", dove il ruolo di superiorità tende al paternalismo, oppure di rivendicazione dal basso che riesce a strappare un privilegio. La gestione dei rapporti di forza in tutte le sue declinazioni, compresa la seduzione e la ossessivizzazione di norme e regolamenti può diventare centrale; nella tensione della conquista del controllo, dall'alto o dal basso, i programmi formali, che esprimono un'intenzione astratta, possono non corrispondere alle strategie e alle tattiche sul campo, alle vittorie e alle sconfitte nei singoli eventi. In uno slogan, ci si concentra sulle battaglie, ma si perde di vista la guerra. 
Il secondo sguardo fonda, invece, la riabilitazione all'interno di una relazione dialogica, che non definisce a priori e per tutti quanto è necessario. Le regole e le prassi sono la base d'appoggio, le prassi minime che permettono una convivenza civile e la cornice entro la quale si decide quale comportamento da seguire è negoziabile e quale no. La focale è aiutare la persona a condividere il valore della regola, il suo senso, ovvero migliorare la convivenza all'interno del contesto.

Si tratta, allora, di una continua e ininterrotta negoziazione, nella tensione di aiutare la persona ad essere realmente adulta e capace di rispettare le regole che garantiscono l'ordine sociale e al contempo di renderla responsabile e capace di offrire all'interno del contesto in cui vive il proprio "personale - unico" contributo. È ovvio come questa tensione possa apparire, a un esame di realtà che consideri le effettive risorse individuali e del microambiente della persona (attraverso un accurato sistema diagnostico multidisciplinare), così come le effettive opportunità del sistema socioeconomico e i valori praticati dalla nostra collettività (non quelli dei discorsi ufficiali, ma quelli che emergono dalle azioni concrete dei leader e del popolo) (Bauman, 2013; Galimberti, 2007), del tutto velleitaria e finzionale. L'impegno profuso per cercare di produrre processi di cambiamento profondo dello stile di vita dei pazienti-detenuti appare sterile, ingenuo, buonista, in sostanza debole. Il salvaguardare spazi e tempi di riflessione può consentire al paziente con un funzionamento antisociale di occupare il territorio, di affermarsi e manovrare a suo piacimento. Riprendendo lo slogan di prima, tenendo fissa la concentrazione sulla guerra, si perdono tutte le battaglie.

\section{L'aggressività}

La gestione della trasgressione alle regole che sfociano in comportamenti aggressivi rientra fra le situazioni più difficili da gestire e trattare. Se, per esempio, il paziente trasgredisce una regola e mette in scena un'aggressione verbale verso un altro detenuto, i quadri interpretativi utilizzati dal sistema di cura e da quello penitenziario saranno diversi.

Per la logica della custodia il comportamento è facilmente riconoscibile e ha un significato univoco ("ti sei comportato in modo aggressivo, ciò conferma l'idea che sei una persona pericolosa") la cui conseguenza è l'espulsione o la minaccia di quest'ultima nel caso di ripetizione del comportamento. Lo scopo sembra essere quello di creare un ambiente controllato, in cui l'aggressività o qualsiasi altro sentimento negativo sarà negato o a malapena sopportato. La logica che potrebbe, invece, essere seguita dall'ottica clinica è quella in cui si interpreta l'aggressione (dal latino aggredior, ad-gradi "movimento verso") come un movimento, seppur primitivo e poco funzionale, che mette in contatto con l'altro e permette di "sentire" un confine fra il Sé e il non-Sé. L'intervento dell'operatore è finalizzato a dare, nell'interazione con i pazienti, un significato del comportamento messo in atto con lo scopo di sviluppare la capacità di mentalizzazione (Allen, Fonagy e Bateman, 2010) e quindi "riflessive", di controllo e di scelta. Se però l'ambiente è percepito come carente di capacità di contenimento, il paziente sperimenterà un crescente distress e attiverà due possibili strategie secondarie:

- disattivante, se crede che ricercare la vicinanza sia inutile: mettono in atto questa strategia i pazienti con un modello di attaccamento distanziante;

- iperattivante, se crede che attuare comportamenti che continuamente sollecitano la vicinanza dia loro maggiori possibilità di ottenerla. Questa strategia è utilizzata da pazienti con un modello di attaccamento invischiato.
Il paziente "disattivante" avrà difficoltà a esprimere le proprie emozioni e non ricercherà la relazione terapeutica: I'intervento non sufficientemente contenitivo avrà determinato un fallimento critico della strategia. I pazienti invischiati utilizzeranno, invece, strategie di ricerca delle relazioni iperattivanti, cercando una distanza con il sistema di cura più vicina rispetto a quella ottimale, manifestando le proprie emozioni in modo da non passare inosservato ed esasperando le situazioni. Anche in questo caso, la mancanza di un intervento sufficientemente contenitivo avrà come conseguenza un'intensificazione degli agiti, invece che uno sviluppo delle capacità di mentalizzazione.

\section{I sentimenti negativi e le angosce di morte}

Pazienti gravi, a volte al limite della trattabilità, come quelli presenti nel contesto carcerario, investono massicciamente tutti coloro che si prendono cura di loro (operatori sanitari e agenti di custodia) di sentimenti di perdita, impotenza e invidia, costringendo gli operatori a un lavoro di elaborazione continua di queste emozioni.

Se il sistema curante non riesce a metabolizzare (Bion, 2016) questi sentimenti si crea una "indigestione" che produce uno stato d'emergenza nel gruppo di lavoro, che riverbera sui pazienti e nel quale si assiste al prevalere del registro corporeo su quello mentale. La ragione viene sostituita dall'istintività e dall'emotività, che hanno reazioni più veloci. L'organismo dell'individuo (dell'operatore!) che si trova in una situazione minacciosa reagisce attivando i sistemi vitali utili per fronteggiare lo stato di pericolo (tachicardia, sudorazione, iperpnea, vasocostrizione, blocco della digestione, ipertensione, iper-reattività); per salvarsi bisogna reagire in fretta, senza riflettere.

Lo stato di emergenza sollecita il gruppo di lavoro, sia esso custodiale che clinico, a un'attivazione emotiva con ipervigilanza, uno stato d'allerta in cui i minimi particolari assumono un valore assoluto e il muoversi per rispondere a una specifica situazione accaduta, anche di modesto rilievo, diventa "questione di vita e di morte". La rappresentazione è che se non si risponde "subito, in modo chiaro e forte", la conseguenza sarà la morte metaforica di qualcuno: l'espulsione del paziente, la sopraffazione di una parte sull'altra, la squalifica di un operatore, lo screditamento di un responsabile.

Non ci si consente l'unica possibilità per ricollegarsi al registro mentale, cioè quella di fermarsi. II fatto di star fermi può portare a far prevalere l'impotenza e l'angoscia di morte. L'azione immediata a una situazione di criticità diventa il modo di governare e di riprendere il controllo su tale angoscia, un modo per dire "sono ancora vivo ed integro".

Purtroppo, però, il potere che si assume nella decisione all'atto è - in quanto meccanismo difensivo dall'angoscia - meramente illusorio e contribuisce a mantenere e perpetuare sia il flusso ininterrotto di eventi e azioni sia lo stato di emergenza. La decisione così presa non risponde a una strategia di pensiero, ma si fonda sulla necessità emotiva di re-azione; la decisione (dal latino: de-caedere = toglier via) non è, infatti, mai qualcosa di spontaneo e istintivo, bensì presuppone un'attenta riflessione in un certo lasso di tempo, unita alla considerazione delle conseguenze della scelta effettuata, così che le parti si riconoscano nelle reciproche differenze e appartenenze.

Un contesto che funziona sullo stato d'allerta emotiva produce, invece, legami di tipo invischiato in cui i confini sfumano. L'effetto è una diffusione dell'identità: gli agenti si trovano a far gli operatori clinici e viceversa, i pazienti a sostenere quali siano "le 
regole di giustizia" che dovrebbero essere applicate, i "sani" appaiono deboli e incapaci, i "malati" forti e con le idee chiare.

\section{Prime conclusioni e scenari possibili}

A meno di non cadere in meccanismi di onnipotenza alimentati da una risposta controtransferale difensiva a quelli di impotenza indotti dai detenuti-pazienti, a noi sembra che, in un contesto così difficile, chiunque - compreso un Dipartimento Dipendenze - abbia difficoltà a mantenere in modo stabile la posizione di "terzo", inteso come portatore di un pensiero in grado di ricomporre le modalità disfunzionali di sistema che si attivano a causa delle specifiche patologie del detenuto-dipendente, del mandato contraddittorio degli agenti e, infine, delle meta-comunicazioni del contesto detentivo ("sei qui perché devi scontare una colpa e quindi la sofferenza che provi non è da alleggerire ma è la giusta componente dell'espiazione").

Una supervisione clinica, a questo punto, diventa strumento necessario e può essere comunque sufficiente a "bonificare" queste dinamiche? O non rischia di diventare essenzialmente un contenitore in cui evacuare le proprie angosce?

II contesto ampio nel quale vengono trattati i dipendenti da sostanze resta, infatti, comunque il carcere: gli operatori entrano al mattino nell'Istituto Penitenziario e vengono sottoposti ai normali controlli degli agenti (riconoscimento dell'identità, perquisizione...); la persona arrestata entra prima di tutto come detenuto e - per quanti sforzi gli operatori possano fare per creare un spazio di cura il più possibile accogliente e favorente la relazione terapeutica - il contesto detentivo più ampio le segnala costantemente che è lì per scontare una pena.

Allora, riprendendo le conclusioni già proposte in un articolo su Animazione Sociale (Bignamini, 2010) per un utilizzo più funzionale delle professionalità e delle competenze di un operatore in carcere, si nota che spesso il terapeuta finisce per "curare il carcere". In altre parole, rassicurare, far decantare, orientare, contenere le istanze distruttive del paziente e del contesto (e le proprie) e dare senso alla relazione perché diventi terapeutica sono tutti procedimenti indispensabili e preliminari che impiegano massicciamente le risorse disponibili per la cura in una misura che è amplificata dall'ambiente carcerario e non dalla patologia.

Le risorse attuali potrebbero essere impiegate, invece, o per aumentare le richieste dei detenuti-pazienti di misure alternative alla pena o per strutturare percorsi che possano favorire il passaggio del paziente dal Tribunale direttamente in comunità senza passare dal contesto detentivo.

Si potrebbe fin d'ora andare in questa direzione:

- perché ci sono gli strumenti tecnici per poter coadiuvare la Magistratura (coadiuvare, non sostituirsi o accogliere deleghe impossibili) nel discernimento fra tossicodipendente che commette reati e delinquente che fa uso di sostanze. La questione non è insuperabile, ma richiede lo sviluppo di una cultura della complessità che orienti a un lavoro di équipe multidisciplinare e multi-istituzionale;

- perché è possibile riorganizzare le risorse attuali dei servizi per le dipendenze operanti in carcere in modo che possano tempestivamente esprimere una valutazione clinica (sempre passibile di revisione per conferma in una fase successiva) prima ancora della traduzione dell'arrestato nella Casa Circondariale;

- perché ci sono le risorse economiche, dato che i costi della comunità sono certamente inferiori ai costi del carcere e che il trasferimento dei tossicodipendenti in comunità territoriali può essere fatto in tempi minori di quelli necessari per costruire nuove strutture carcerarie. Il problema si pone, come emerso anche dal lavoro del Tavolo 4 "Minorità sociale, vulnerabilità, dipendenze" degli Stati Generali dell'Esecuzione Penale (Ministero della Giustizia, 2016), non sul piano delle risorse in termini assoluti, ma in relazione alla separazione dei bilanci, alla compartimentazione tra Sanità, Sociale e Giustizia, che impedisce un uso razionale ed efficiente delle risorse stesse;

- perché lo sviluppo delle comunità produrrebbe effetti positivi: a) sul piano terapeutico (compatibilità degli interventi con quelli previsti dai servizi per le dipendenze, quindi reale coerenza e continuità nella cura prima-durante-dopo la limitazione della libertà); b) sul piano culturale e normativo (sottolineerebbe che la tossicodipendenza è una patologia e non un crimine); c) sul piano sociale (valorizzerebbe una progettualità sociale di cui le comunità terapeutiche sono portatrici).

In questo senso e in linea con quanto appena proposto - adeguatamente esplorato sul piano teorico, della fattibilità e quindi declinato - ci si augura che le difficoltà che oggettivamente si riscontrano nel tentare un approccio terapeutico nel contesto carcerario attuale, invece di essere semplicemente negate, o magari scisse attraverso la squalifica di chi tenta di gestire elevate complessità, possano essere riconosciute e comprese, e portino un frutto creativo e fecondo, ispiratore di nuovi pensieri.

\section{Bibliografia}

Allen J.G., Fonagy P., Bateman A.W. (2010). La mentalizzazione nella pratica clinica. Milano: Raffaello Cortina Editore.

Bauman Z. (2013). Le sorgenti del male. Trento: Erickson.

Bignamini E. (2010). Distinguere il tossicodipendente dal delinquente. Ipotesi per incrementare spazi di cura. Animazione Sociale, 60-70.

Bion W.R. (2016). Esperienze nei gruppi. Roma: Armando Editore.

Bowlby J. (1996). Una base sicura. Applicazioni cliniche della teoria dell'attaccamento. Milano: Raffaello Cortina Editore.

Ferrero A. (1995). Note sul rapporto tra identità, stile di vita e vicende familiari interiorizzate. In: Mascetti A., Zighetti A. (a cura di), La costellazione familiare. Torino: Stampa Brunello.

Foucault M. (1976). Sorvegliare e punire. Torino: Einaudi.

Galimberti U. (2007). L'ospite inquietante. Il nichilismo e i giovani. Milano: Feltrinelli.

Liotti G., Farina B. (2011). Sviluppi traumatici. Eziopatogenesi, clinica e terapia della dimensione dissociativa. Milano: Raffaello Cortina Editore.

Ministero della Giustizia (2016). Tavolo 4 "Minorità sociale, vulnerabilità, dipendenze" degli Stati Generali dell'Esecuzione Penale, www.giustizia.it/giustizia/it/mg_2_19_1_4.page, 5 febbraio 2016.

Piaget J. (1972). Il giudizio morale nel fanciullo. Firenze: Giunti Editore. Winnicott D.W. (2005). Sviluppo affettivo e ambiente. Roma: Armando Editore. 